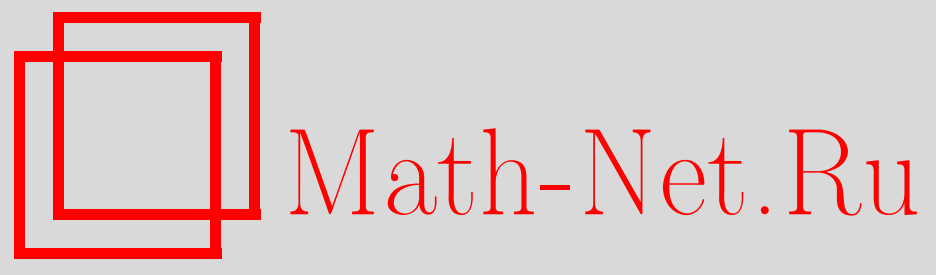

K. Шимитерна, Д. Леви, Интегрируемость дифференциально-разностных уравнений с дискретными кинками, $T M \Phi, 2011$, том 167, номер 3, 496-513

DOI: https://doi.org/10.4213/tmf6657

Использование Общероссийского математического портала Math-Net.Ru подразумевает, что вы прочитали и согласны с пользовательским соглашением http://www.mathnet.ru/rus/agreement

Параметры загрузки:

IP: 18.207.199.55

26 апреля 2023 г., 17:58:07

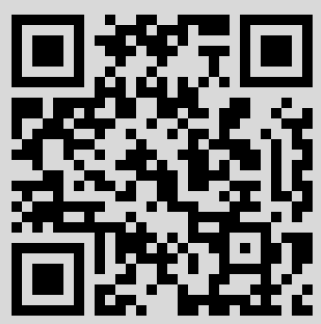




\title{
ИНТЕГРИРУЕМОСТЬ ДИФФЕРЕНЦИАЛЬНО-РАЗНОСТНЫХ УРАВНЕНИЙ С ДИСКРЕТНЫМИ КИНКАМИ
}

\begin{abstract}
Обсуждается ряд введенных Барашенковым, Окстоби и Пелиновски моделей, описывающих некоторые дискретные аппроксимации $\phi^{4}$-теории, сохраняющие бегущие кинковые решения. С помощью многомасштабного теста показано, что они обладают определенными свойствами интегрируемости, поскольку для них выполнены условия $A_{1}$ - и $A_{2}$-интегрируемости. Однако они не являются интегрируемыми, поскольку не удовлетворяют условиям $A_{3}$-интегрируемости.
\end{abstract}

Ключевые слова: решеточные уравнения, кинковые решения, многомасштабное разложение, интегрируемые уравнения.

\section{1. ВВЕДЕНИЕ}

В работе [1] рассмотрены исключительные дискретизации $\phi^{4}$-теории, в которых стационарный кинк может располагаться в произвольных точках между узлами решетки. Требование, чтобы кинк являлся трансляционно-инвариантным, порождает три семейства исключительных дискретизаций, дающих новые дифференциальноразностные уравнения, интегрируемость которых не установлена. Сама по себе непрерывная $\phi^{4}$-модель не является полностью интегрируемой, т.е. не обладает бесконечным числом обобщенных симметрий, законов сохранения и точных решений, однако представляет собой аппроксимацию многих $C$ - или $S$-интегрируемых нелинейных моделей, таких как уравнение Лиувилля или уравнение синус-Гордон, поэтому анализ их интегрируемости представляет собой интересную задачу. Мы применяем для этого многомасштабный анализ нелинейных дискретных уравнений, поскольку он позволяет провести тест на интегрируемость и выразить степень интегрируемости уравнений.

В разделах 2 и 3 представлен обзор моделей, предложенных в работе [1], и результатов, необходимых для применения к ним многомасштабного анализа. В разделе 4 приведены результаты вычислений, а в разделе 5 даны некоторые заключительные замечания. Подробности вычислений вынесены в приложение.

* Dipartimento di Ingegneria Elettronica, Università di Roma Tre; INFN, Sezione di Roma Tre, Roma, Italy. E-mail: scimiterna@fis.uniroma3.it, levi@roma3.infn.it 


\section{2. ИСКЛЮЧИТЕЛЬНЫЕ МОДЕЛИ}

$\phi^{4}$-Теория представляет собой одну из наиболее важных нелинейных моделей для описания систем статистической механики и теории поля [2], [3]. Дискретным аналогом $\phi^{4}$-кинков являются решения уравнений вида

$$
\ddot{u}_{n}=\frac{u_{n+1}-2 u_{n}+u_{n-1}}{h^{2}}+\frac{u_{n}}{2}-Q_{n}\left(u_{n-1}, u_{n}, u_{n+1}\right) \text {, }
$$

где $u_{n}=u(h n, t)$ - шаг решетки, $h$ - константа. Функция $Q_{n}$ выбрана так, чтобы в непрерывном пределе в первом порядке по $h$ получался $\phi^{4}$-потенциал $Q=-u^{3} / 2$. Эти кинки используются для описания волн зарядовой плотности в полимерах и металлах [4], узких доменных стенок в сегнетоэлектриках [5], несоизмеримости в диэлектрических кристаллах [6], а также топологических возбуждений в цепочках с водородной связью [7], [8]. С физической точки зрения одним из наиболее важных свойств доменных стенок и топологических дефектов является их подвижность [9]. Известно, что дискретные уравнения (1) допускают стационарные кинковые решения [9], [10], однако вопрос, существуют ли бегущие дискретные кинки, остается открытым [11]-[16]. Дискретизация нарушает лоренцеву инвариантность, и существование бегущих дискретных кинков становится нетривиальным. Действительно, если уравнение не является автономным, то дискретизация нарушает даже трансляционную инвариантность уравнений (1) из-за присутствия барьера Пайерлса-Набарро - дополнительного периодического потенциала, порожденного дискретностью. Удивительно, что имеются несколько исключительных дискретизаций, которые, несмотря на нарушение трансляционной инвариантности уравнения, допускают существование трансляционно-инвариантных кинков, т. е. кинков, центры которых расположены в произвольных точках между узлами. Одна из таких дискретизаций была открыта в работах [14], [15], где использовались соображения, связанные с минимальностью энергии Богомольного. В работе [1] проведено систематическое исследование этих исключительных случаев, основанное на наблюдении, что из трансляционной инвариантности кинка следует существование лежащего в его основе одномерного отображения $u_{n+1}=F\left(u_{n}\right)$. Простой алгоритм, основанный на этом наблюдении, дает три класса исключительных дискретизаций:

$$
\begin{aligned}
Q_{1} \doteq & \frac{1}{20}\left[\mu_{1}\left(u_{n+1}+u_{n-1}\right)\left(u_{n+1}^{2}+u_{n-1}^{2}\right)+\right. \\
& \left.+\mu_{3} u_{n}^{2}\left(u_{n+1}+u_{n-1}\right)+\mu_{2} u_{n}\left(u_{n+1}^{2}+u_{n}^{2}+u_{n-1}^{2}+u_{n+1} u_{n-1}\right)\right], \\
Q_{2} \doteq & \mu_{1}^{2}\left(u_{n+1}^{3}+u_{n-1}^{3}\right)+2 \mu_{3}\left(\mu_{1}-\mu_{2}\right) u_{n+1} u_{n} u_{n-1}+ \\
& +\mu_{1}\left(\mu_{1}-\mu_{2}\right) u_{n+1} u_{n-1}\left(u_{n+1}+u_{n-1}\right)+\mu_{1}\left(2 \mu_{3}+\mu_{2}\right) u_{n}\left(u_{n+1}^{2}+u_{n-1}^{2}\right)+ \\
& +2 \mu_{1}\left(\mu_{2}+\mu_{3}\right) u_{n}^{3}+\left[2 \mu_{1}^{2}+\mu_{3}^{2}+\mu_{2}\left(\mu_{3}-\mu_{1}\right)\right] u_{n}^{2}\left(u_{n+1}+u_{n-1}\right), \\
Q_{3} \doteq & \mu_{1}\left(1+\frac{h^{2}}{4}\right) u_{n}\left(u_{n+1}+u_{n-1}\right)^{2}-2 \mu_{1} u_{n-1} u_{n+1}\left(u_{n+1}+u_{n-1}\right)+ \\
& +\left(\frac{1}{4}-\frac{\mu_{2}}{2}\right) u_{n}^{2}\left(u_{n+1}+u_{n-1}\right)+\mu_{2} u_{n-1} u_{n} u_{n+1},
\end{aligned}
$$

где в первых двух уравнениях, как указано в работе [1], коэффициенты $\mu_{1}, \mu_{2}, \mu_{3}$ не являются независимыми.

6 Теоретическая и математическая физика, т. 167, № 3, 2011 г. 


\section{3. МНОГОМАСШТАБНЫЕ РАЗЛОЖЕНИЯ РЕШЕТОЧНЫХ УРАВНЕНИЙ}

Для полноты мы приводим здесь основные идеи многомасштабного разложения для решеточных уравнений, изложенные в работе [17] в случае разложений в терминах аналитических функций.

\section{1. Разложение вещественных дисперсионных уравнений в частных производных.}

3.1.1. От сдвигов к производным. Рассмотрим функцию $u_{n}: \mathbb{Z} \rightarrow \mathbb{R}$, зависящую от дискретного индекса $n \in \mathbb{Z}$, и предположим, что:

а) зависимость функции $u_{n}$ от $n$ реализуется через медленную переменную $n_{1} \doteq$ $\varepsilon n \in \mathbb{R}, \varepsilon \in \mathbb{R}, 0<\varepsilon \ll 1$, т. е. $u_{n} \doteq u\left(n_{1}\right)$;

б) $n_{1}$ изменяется в той области на целочисленной оси, в которой функция $u\left(n_{1}\right)$ аналитична;

в) радиус сходимости ряда Тейлора по $n_{1}$ достаточно широк, чтобы включать в качестве внутренних точек все точки, задействованные в дискретном уравнении.

С учетом этих гипотез можно записать действие оператора сдвига $T_{n}$, определяемого как $T_{n} u_{n} \doteq u_{n+1}=u\left(n_{1}+\varepsilon\right)$ для переменной $n_{1}$, в виде

$$
\begin{aligned}
T_{n} u\left(n_{1}\right) & =u\left(n_{1}\right)+\varepsilon u^{(1)}\left(n_{1}\right)+\frac{\varepsilon^{2}}{2} u^{(2)}\left(n_{1}\right)+\cdots+\frac{\varepsilon^{i}}{i !} u^{(i)}\left(n_{1}\right)+\cdots= \\
& =\sum_{i=0}^{\infty} \frac{\varepsilon^{i}}{i !} u^{(i)}\left(n_{1}\right),
\end{aligned}
$$

где $u^{(i)}\left(n_{1}\right) \doteq \frac{d^{i} u\left(n_{1}\right)}{d n_{1}^{i}} \doteq d_{n_{1}}^{i} u\left(n_{1}\right), d_{n_{1}}$ - оператор полной производной. Из формулы (3) следует, что можно записать следующее формальное разложение для оператора сдвига $T_{n}$ :

$$
T_{n}=\sum_{i=0}^{\infty} \frac{\varepsilon^{i}}{i !} d_{n_{1}}^{i} \doteq e^{\varepsilon d_{n_{1}}} .
$$

Если $u_{n}$ зависит одновременно от бъстрой переменной $n$ и от медленной переменной $n_{1}$, т. е. $u_{n} \doteq u\left(n, n_{1}\right)$, то действие полного оператора сдвига $T_{n}$ даст $T_{n} u_{n} \doteq u_{n+1}=u\left(n+1, n_{1}+\varepsilon\right)$. Поэтому можно разбить его на части, соответствующие частным операторам сдвига $\mathcal{T}_{n}$ и $\mathcal{T}_{n_{1}}^{(\varepsilon)}$, которые определяются соответственно как $\mathcal{T}_{n} u\left(n, n_{1}\right)=u\left(n+1, n_{1}\right)$ и $\mathcal{T}_{n_{1}}^{(\varepsilon)} u\left(n, n_{1}\right)=u\left(n, n_{1}+\varepsilon\right), T_{n} \doteq \mathcal{T}_{n} \mathcal{T}_{n_{1}}^{(\varepsilon)}$, где $\mathcal{T}_{n_{1}}^{(\varepsilon)}$ также задается соотношением (4). Зависимость $u_{n}$ от $n$ легко продолжить на случай одной быстрой переменной $n$ и $K$ медленных переменных $n_{j} \doteq \varepsilon_{j} n, \varepsilon_{j} \in \mathbb{R}, 1 \leqslant j \leqslant K$. Полный оператор сдвига $T_{n}$ теперь запишется как $T_{n} \doteq \mathcal{T}_{n} \prod_{j=1}^{K} \mathcal{T}_{n_{j}}^{\left(\varepsilon_{j}\right)}$.

Рассмотрим нелинейное разностное уравнение в частных производных

$$
F\left[u_{\{n+i\}_{i=-\mathcal{N}^{(-)}}^{\mathcal{N}^{(+)}},\{m+j\}_{j=-\mathcal{M}^{(-)}}^{\mathcal{M}^{(+)}}}\right]=0, \quad \mathcal{N}^{( \pm)}, \mathcal{M}^{( \pm)} \geqslant 0
$$

для функции $u_{n, m}: \mathbb{Z}^{2} \rightarrow \mathbb{R}$, зависящей от двух целочисленных индексов $n$ и $m$, которые мы будем называть соответственно пространственной и временно́й переменными. Уравнение (5) содержит $m$-сдвиги в интервале $\left(m-\mathcal{M}^{(-)}, m+\mathcal{M}^{(+)}\right)$ 
и $n$-сдвиги в интервале $\left(n-\mathcal{N}^{(-)}, n+\mathcal{N}^{(+)}\right)$. В рамках гипотез а-в можно дать представление сдвинутых значений функции $u_{n, m}$ в виде ряда в окрестности точки $(n, m)$. Выбирая

$$
\varepsilon_{n_{1}} \doteq N_{1} \varepsilon, \quad \varepsilon_{m_{j}} \doteq M_{j} \varepsilon^{j}, \quad 1 \leqslant j \leqslant K, \quad \varepsilon, N_{1}, M_{j} \in \mathbb{R}
$$

можем записать

$$
\begin{aligned}
T_{n} & =\mathcal{T}_{n} \mathcal{T}_{n_{1}}^{\left(\varepsilon_{n_{1}}\right)}=\mathcal{T}_{n} \sum_{j=0}^{\infty} \varepsilon^{j} \mathcal{A}_{n}^{(j)}, \quad \mathcal{A}_{n}^{(j)} \doteq \frac{N_{1}^{j}}{j !} \partial_{n_{1}}^{j} \\
T_{m} & =\mathcal{T}_{m} \prod_{j=1}^{K} \mathcal{T}_{m_{j}}^{\left(\varepsilon_{m_{j}}\right)}=\mathcal{T}_{m} \sum_{j=0}^{\infty} \varepsilon^{j} \mathcal{A}_{m}^{(j)} \\
T_{n} T_{m} & =\mathcal{T}_{n} \mathcal{T}_{m} \mathcal{T}_{n_{1}}^{\left(\varepsilon_{n_{1}}\right)} \prod_{j=1}^{K} \mathcal{T}_{m_{j}}^{\left(\varepsilon_{m_{j}}\right)}=\mathcal{T}_{n} \mathcal{T}_{m} \sum_{j=0}^{\infty} \varepsilon^{j} \mathcal{A}_{n, m}^{(j)}
\end{aligned}
$$

где операторы $\mathcal{A}_{m}^{(j)}, \mathcal{A}_{n, m}^{(j)}$ представляют собой подходящие комбинации операторов $\mathcal{A}_{m_{k}}^{(j)} \doteq \frac{M_{k}^{j}}{j !} \partial_{m_{k}}^{j}$ и $\mathcal{A}_{n}^{(j)} \doteq \frac{N_{1}^{j}}{j !} \partial_{n_{1}}^{j}$ (подробнее см. [18]). При подстановке явных выражений (6) для операторов сдвига в члены с производными по медленным переменным в уравнении (5) мы получаем зависящее от $\varepsilon$ дифференциальное уравнение в частных производных (ДУЧП) бесконечного порядка. Теперь предположим, что для функции $u=u\left(n, m, n_{1},\left\{m_{j}\right\}_{j=1}^{K}, \varepsilon\right)$ имеется двойное разложение по гармоникам и пертурбативному параметру $\varepsilon$ :

$$
\begin{gathered}
u\left(n, m, n_{1},\left\{m_{j}\right\}_{j=1}^{K}, \varepsilon\right)=\sum_{\gamma=1}^{\infty} \sum_{\alpha=-\gamma}^{\gamma} \varepsilon^{\gamma} u_{\gamma}^{(\alpha)}\left(n_{1},\left\{m_{j}\right\}_{j=1}^{K}\right) E_{n, m}^{\alpha}, \\
E_{n, m} \doteq e^{i[\kappa n-\omega(\kappa) m]}, \quad u_{\gamma}^{(-\alpha)}=\bar{u}_{\gamma}^{(\alpha)}
\end{gathered}
$$

где индекс $\gamma$ выбирается согласно условию $\gamma \geqslant 1$, чтобы позволить любой нелинейной части уравнения (5) входить как возмущение в многомасштабное разложение.

3.1.2. От производных к сдвигам. На основе разбиения (5) в соответствии с различными степенями $\varepsilon$ и различными гармониками многомасштабный подход позволяет получить из заданного разностного уравнения в частных производных ДУЧП для амплитуд $u_{\gamma}^{(\alpha)}$. Исходя из полученного ДУЧП можно записать разностное уравнение в частных производных, обращая выражение для оператора сдвига:

$$
\partial_{n_{1}}=\ln \mathcal{T}_{n_{1}}=\ln \left(1+h_{n_{1}} \Delta_{n_{1}}^{(+)}\right) \doteq \sum_{i=1}^{\infty} \frac{(-1)^{i-1} h_{n_{1}}^{i}}{i} \Delta_{n_{1}}^{(+) i}
$$

где $\Delta_{n_{1}}^{(+)} \doteq\left(\mathcal{T}_{n_{1}}-1\right) / h_{n_{1}}-$ просто первый разностный оператор сдвига "вперед" по медленной переменной $n_{1}$.

Только когда мы потребуем, чтобы функция $u_{n}$ была медленно меняющейся функцией порядка $l$ по $n_{1}$, т. е. $\Delta_{n_{1}}^{\ell+1} u_{n} \approx 0$, оператор $\partial_{n_{1}}$ редуцируется к многочленам по $\Delta_{n_{1}}$ порядка, не превышающего $l$. В работе [19] для индексов $n_{1}$ и $m_{1}$ 
было выбрано значение $l=2$, а для индекса $m_{2}$ - значение $l=1$, и было показано, что интегрируемое уравнение с решеточным потенциалом Кортевега-де Фриза $(\mathrm{K} \Phi)[20]$ редуцируется к полностью дискретному и локальному нелинейному уравнению Шредингера (НУШ), для которого было установлено, что оно не является интегрируемым вследствие сингулярности конфайнмента и алгебраической энтропии [21], [22]. Следовательно, если переходить от производных к сдвигам, то в итоге получается, вообще говоря, нелокальное разностное уравнение в частных производных для медленных переменных $n_{\kappa}$ и $m_{\delta}$.

3.2. Порядки за рамками НУШ и условия интегрируемости. Многомасштабное разложение разностного уравнения (5) для аналитических функций будет порождать непрерывное ДУЧП. Поэтому многомасштабный тест на интегрируемость требует, чтобы уравнение типа (1) было интегрируемым, если его многомасштабное разложение переходит в иерархию НУШ. Для этого нужно рассмотреть более высокие порядки, чем тот, для которого получается (интегрируемое) НУШ для гармонических $u_{1}^{(1)}$. Первая попытка выйти за рамки порядка, задаваемого НУШ, была предпринята авторами работ [23], [24]. На основе $S$-интегрируемых моделей (т. е. моделей, интегрируемых преобразованием рассеяния) с помощью комбинации асимптотического функционального анализа и спектральных методов им удалось удалить все секулярные члены из редуцированных уравнений порядок за порядком. Результаты можно кратко сформулировать следующим образом.

1. Число медленных временны́х переменных, требуемых для амплитуд $u_{j}^{(\alpha)}$, совпадает с числом ненулевых коэффициентов $\omega_{j}(\kappa)=\frac{1}{j !} \frac{d^{j} \omega(k)}{d k^{j}}$.

2. Амплитуда $u_{1}^{(1)}$ эволюционирует по медленным временам $t_{\sigma}, \sigma \geqslant 3$, в соответствии с $\sigma$-м уравнением иерархии НУШ.

3. Амплитуды высших возмущений первой гармоники $u_{j}^{(1)}, j \geqslant 2$, с учетом определенных асимптотических граничных условий эволюционируют по медленным временам $t_{\sigma}, \sigma \geqslant 2$, в соответствии с некоторыми линейными неоднородными уравнениями.

Таким образом, сокращение всех секулярных членов на каждом этапе процесса возмущения является достаточным условием для того, чтобы единственным образом фиксировать эволюционные уравнения, которым подчиняется каждая амплитуда $u_{j}^{(1)}, j \geqslant 1$, для каждого медленного времени $t_{\sigma}$. Из сформулированного выше результата 2 следует, что иерархия интегрируемых уравнений всегда обеспечивает совместные эволюции для единой функции $u$, зависящей от различных времен, т. е. уравнения в иерархии представляют собой обобщенные симметрии друг для друга. Таким образом, данная процедура обеспечивает необходимое и достаточное условия для получения редуцированных уравнений, свободных от секулярных членов [23].

Далее, следуя работам [25], введем

ОПРЕДЕЛЕНИЕ 1. Говорят, что нелинейное ДУЧП интегрируемо, если оно обладает нетривиальной парой Лакса и, следовательно, бесконечным числом обобщенных симметрий.

Как следствие этого определения имеем следующее 
ПреДЛОЖЕНИЕ 1. Если уравнение (5) является $S$-интегрируемым, то при многомасштабном разложении функции $u_{j}^{(1)}, j \geqslant 1$, удовлетворяют уравнениям

$$
\begin{gathered}
\partial_{t_{\sigma}} u_{1}^{(1)}=K_{\sigma}\left[u_{1}^{(1)}\right], \\
M_{\sigma} u_{j}^{(1)}=f_{\sigma}(j), \quad M_{\sigma} \doteq \partial_{t_{\sigma}}-K_{\sigma}^{\prime}\left[u_{1}^{(1)}\right]
\end{gathered}
$$

для всех $\sigma \geqslant 2$, где $K_{\sigma}\left[u_{1}^{(1)}\right]-\sigma$-й поток в иерархии НУШ. Все остальные функиии $u_{j}^{(\kappa)}, \kappa \geqslant 2$, выражантся в терминах дифференииальных мономов по $u_{\rho}^{(1)}, \rho \leqslant j$.

В формулах (9б) $f_{\sigma}(j)$ - неоднородный нелинейный вынуждающий член, а $K_{\sigma}^{\prime}[u] v-$ производная Фреше нелинейного члена $K_{\sigma}[u]$ по направлению $v: K_{\sigma}^{\prime}[u] v \doteq$ $\left.\frac{d}{d s} K_{\sigma}[u+s v]\right|_{s=0}$, т. е. линеаризация $K_{\sigma}[u]$ по направлению $v$. Уравнения (9) представляют собой необходимое условие интегрируемости и являются иерархией совместных эволюций для той же функции $u_{1}^{(1)}$ по различным медленным временам. Совместность уравнений (9б) не всегда гарантирована, но зависит от условий типа коммутативности между членами $f_{\sigma}(j)$ в их правых частях. Тогда легко доказать, что операторы $M_{\sigma}$, определенные в (9б), коммутируют между собой. Коль скоро зафиксирован индекс $j \geqslant 2$ в системе уравнений (9б), из этого условия коммутативности вытекают условия совместности

$$
M_{\sigma} f_{\sigma^{\prime}}(j)=M_{\sigma^{\prime}} f_{\sigma}(j) \quad \forall \sigma, \sigma^{\prime} \geqslant 2,
$$

где, поскольку $f_{\sigma}(j)$ и $f_{\sigma^{\prime}}(j)$ являются функциями различных возмущений фундаментальных гармоник вплоть до степени $j-1$, производные по времени $\partial_{t_{\sigma}}$ и $\partial_{t_{\sigma^{\prime}}}$ этих гармоник, появляющиеся соответственно в $M_{\sigma}$ и $M_{\sigma^{\prime}}$, должны быть исключены с помощью эволюционных уравнений (9) вплоть до индекса $j-1$. Условия коммутативности (10) оказываются тестом на интегрируемостъ.

Следуя работе [23], выскажем гипотезу, что соотношения (9) являются достаточным условием интегрируемости или что интегрируемость является необходимым условием для многомасштабного разложения, если выполнены уравнения (9). Чтобы построить функции $f_{\sigma}(j)$, в соответствии с предложением 1 введем следующие определения.

ОПреДЕЛЕНиЕ 2. Дифференциальный моном $\rho\left[u_{j}^{(1)}\right], j \geqslant 1$, по функциям $u_{j}^{(1)}$, его комплексным сопряжениям и его $\xi$-производным является мономом "калибров$\kappa u ” 1$, если он обладает свойством преобразования

$$
\rho\left[\tilde{u}_{j}^{(1)}\right]=e^{i \theta} \rho\left[u_{j}^{(1)}\right], \quad \tilde{u}_{j}^{(1)} \doteq e^{i \theta} u_{j}^{(1)} .
$$

ОПРЕДЕЛЕНИЕ 3. Конечномерное векторное пространство $\mathcal{P}_{n}, n \geqslant 2$, представляет собой набор всех дифференциальных многочленов по функциям $u_{j}^{(1)}, j \geqslant 1$, их комплексным сопряжениям и их $\xi$-производным порядка $n$ по $\varepsilon$ калибровки 1 , если

$$
\operatorname{order}\left(\partial_{\xi}^{m} u_{j}^{(1)}\right)=\operatorname{order}\left(\partial_{\xi}^{m} \bar{u}_{j}^{(1)}\right)=m+j, \quad m \geqslant 0 .
$$

ОПРЕДЕЛЕНИЕ 4. Пространство $\mathcal{P}_{n}(m)$ при $m \geqslant 1$ и $n \geqslant 2$ является подпространством в $\mathcal{P}_{n}$, элементы которого суть дифференциальные многочлены по функциям $u_{j}^{(1)}$, их комплексным сопряжениям и их $\xi$-производным порядка $n$ по $\varepsilon$ калибровки 1 при $1 \leqslant j \leqslant m$. 
Из определения 4 можно видеть, что, вообще говоря, $K_{\sigma}\left[u_{1}^{(1)}\right] \in \partial_{\xi}^{\sigma} u_{1}^{(1)} \cup \mathcal{P}_{\sigma+1}(1)$ и $f_{\sigma}(j) \in \mathcal{P}_{j+\sigma}(j-1)$, где $j, \sigma \geqslant 2$. Базисные мономы пространства $\mathcal{P}_{n}(m)$ можно найти, например, в работе [18].

ПрЕДЛОЖЕНИЕ 2. Если для каждого фиксированного $j \geqslant 2$ выполнено уравнение (10) при $\sigma=2$ и $\sigma^{\prime}=3$, а именно $M_{2} f_{3}(j)=M_{3} f_{2}(j)$, то для любого $\sigma \geqslant 4$ существуют единственные дифференииальные многочлены $f_{\sigma}(j)$ такие, что потоки $M_{\sigma} u_{j}^{(1)}=f_{\sigma}(j)$ коммутируют при любъх $\sigma \geqslant 2$.

Таким образом, среди соотношений (10) следует протестировать только соотношения с $\sigma=2$ и $\sigma^{\prime}=2$.

ПрЕДЛОЖЕНИЕ 3. Однородное уравнение $M_{\sigma} u=0$ не имеет решения $и$ в векторном пространстве $\mathcal{P}_{m}$, m. е. $\operatorname{Ker}\left(M_{\sigma}\right) \cap \mathcal{P}_{m}=\varnothing$.

Следовательно, многомасштабное разложение (9) является свободным от секулярных членов. Наконец, определим степень интегрируемости заданного уравнения.

ОПРЕДЕЛЕНИЕ 5. Если соотношения (10) выполнены вплоть до индекса $j, j \geqslant 2$, то будем говорить, что наше уравнение является асимптотически интегрируемым со степенъю $j$, или $A_{j}$-интегрируемым.

Условия интегрируемости для иерархии НУШ. Укажем условия асимптотической интегрируемости порядка $k$ или условия $A_{k}$-интегрируемости. Для упрощения обозначений будем использовать для $u_{j}^{(1)}$ краткую запись $u(j)$. Для удобства читателя выпишем также потоки $K_{\sigma}[u]$ иерархии НУШ для $u$ вплоть до $\sigma=4$ :

$$
\begin{gathered}
K_{1}[u] \doteq A u_{\xi}, \\
K_{2}[u] \doteq-i \rho_{1}\left[u_{\xi \xi}+\frac{\rho_{2}}{\rho_{1}}|u|^{2} u\right] \\
K_{3}[u] \doteq B\left[u_{\xi \xi \xi}+\frac{3 \rho_{2}}{\rho_{1}}|u|^{2} u_{\xi}\right], \\
K_{4}[u] \doteq-i C\left\{u_{\xi \xi \xi \xi}+\frac{\rho_{2}}{\rho_{1}}\left[\frac{3 \rho_{2}}{2 \rho_{1}}|u|^{4} u+4|u|^{2} u_{\xi \xi}+3 u_{\xi}^{2} \bar{u}+2\left|u_{\xi}\right|^{2} u+u^{2} \bar{u}_{\xi \xi}\right]\right\},
\end{gathered}
$$

где $\rho_{1}, \rho_{2}, A, B$ и $C$ - произвольные комплексные постоянные.

Условие $A_{1}$-интегрируемости определяется вещественностью коэффициента $\rho_{2}$ при нелинейном члене в НУШ.

Условия $A_{2}$-интегрируемости получаются при выборе $j=2$ в условиях совместности уравнений (10) при $\sigma=2$ и $\sigma^{\prime}=3$ :

$$
M_{2} f_{3}(j)=M_{3} f_{2}(j) .
$$

В этом случае имеем $f_{2}(2) \in \mathcal{P}_{4}(1)$ и $f_{3}(2) \in \mathcal{P}_{5}(1)$ при $\operatorname{dim}\left(\mathcal{P}_{4}(1)\right)=2$ и $\operatorname{dim}\left(\mathcal{P}_{5}(1)\right)=$ 5 , так что $f_{2}(2)$ и $f_{3}(2)$ соответственно задаются двумя и пятью комплексными постоянными:

$$
\begin{aligned}
f_{2}(2) \doteq & a u_{\xi}(1)|u(1)|^{2}+b \bar{u}_{\xi}(1) u(1)^{2} \\
f_{3}(2) \doteq & \alpha|u(1)|^{4} u(1)+\beta\left|u_{\xi}(1)\right|^{2} u(1)+\gamma u_{\xi}(1)^{2} \bar{u}(1)+ \\
& +\delta \bar{u}_{\xi \xi}(1) u(1)^{2}+\varepsilon|u(1)|^{2} u_{\xi \xi}(1) .
\end{aligned}
$$


Действуя таким образом, при $\rho_{2} \neq 0$ исключая из уравнения (12) производные $u(1)$ по медленным временам $t_{2}$ и $t_{3}$, используя эволюции (9a) при $\sigma=2$ и $\sigma^{\prime}=3$ и почленно приравнивая, получаем условия $A_{2}$-интегрируемости:

$$
a=\bar{a}, \quad b=\bar{b} .
$$

На данном этапе имеются два условия, получаемые из требования вещественности коэффициентов $a$ и $b$. Постоянные $\alpha, \beta, \gamma, \delta$ выражаются через $a$ и $b$ следующим образом:

$$
\alpha=\frac{3 i B \rho_{2} a}{4 \rho_{1}^{2}}, \quad \beta=\frac{3 i B b}{\rho_{1}}, \quad \gamma=\frac{3 i B a}{2 \rho_{1}}, \quad \delta=0, \quad \varepsilon=\gamma .
$$

Условия $A_{3}$-интегрируемости выводятся аналогично, если в уравнении (12) положить $j=3$. При этом имеем $f_{2}(3) \in \mathcal{P}_{5}(2)$ и $f_{3}(3) \in \mathcal{P}_{6}(2)$, где $\operatorname{dim}\left(\mathcal{P}_{5}(2)\right)=12$ и $\operatorname{dim}\left(\mathcal{P}_{6}(2)\right)=26$, так что $f_{2}(3)$ и $f_{3}(3)$ задаются соответственно с помощью 12 и 26 комплексных постоянных:

$$
\begin{aligned}
f_{2}(3) \doteq & \tau_{1}|u(1)|^{4} u(1)+\tau_{2}\left|u_{\xi}(1)\right|^{2} u(1)+\tau_{3}|u(1)|^{2} u_{\xi \xi}(1)+\tau_{4} \bar{u}_{\xi \xi}(1) u(1)^{2}+ \\
& +\tau_{5} u_{\xi}(1)^{2} \bar{u}(1)+\tau_{6} u_{\xi}(2)|u(1)|^{2}+\tau_{7} \bar{u}_{\xi}(2) u(1)^{2}+\tau_{8} u(2)^{2} \bar{u}(1)+ \\
& +\tau_{9}|u(2)|^{2} u(1)+\tau_{10} u(2) u_{\xi}(1) \bar{u}(1)+\tau_{11} u(2) \bar{u}_{\xi}(1) u(1)+\tau_{12} \bar{u}(2) u_{\xi}(1) u(1), \\
f_{3}(3) \doteq & \gamma_{1}|u(1)|^{4} u_{\xi}(1)+\gamma_{2}|u(1)|^{2} u(1)^{2} \bar{u}_{\xi}(1)+\gamma_{3}|u(1)|^{2} u_{\xi \xi \xi}(1)+\gamma_{4} u(1)^{2} \bar{u}_{\xi \xi \xi}(1)+ \\
& +\gamma_{5}\left|u_{\xi}(1)\right|^{2} u_{\xi}(1)+\gamma_{6} \bar{u}_{\xi \xi}(1) u_{\xi}(1) u(1)+\gamma_{7} u_{\xi \xi}(1) \bar{u}_{\xi}(1) u(1)+ \\
& +\gamma_{8} u_{\xi \xi}(1) u_{\xi}(1) \bar{u}(1)+\gamma_{9}|u(1)|^{4} u(2)+\gamma_{10}|u(1)|^{2} u(1)^{2} \bar{u}(2)+\gamma_{11} \bar{u}_{\xi}(1) u(2)^{2}+ \\
& +\gamma_{12} u_{\xi}(1)|u(2)|^{2}+\gamma_{13}\left|u_{\xi}(1)\right|^{2} u(2)+\gamma_{14}|u(2)|^{2} u(2)+\gamma_{15} u_{\xi}(1)^{2} \bar{u}(2)+ \\
& +\gamma_{16}|u(1)|^{2} u_{\xi \xi}(2)+\gamma_{17} u(1)^{2} \bar{u}_{\xi \xi}(2)+\gamma_{18} u(2) \bar{u}_{\xi \xi}(1) u(1)+\gamma_{19} u(2) u_{\xi \xi}(1) \bar{u}(1)+ \\
& +\gamma_{20} \bar{u}(2) u_{\xi \xi}(1) u(1)+\gamma_{21} u(2) u_{\xi}(2) \bar{u}(1)+\gamma_{22} \bar{u}(2) u_{\xi}(2) u(1)+ \\
& +\gamma_{23} u_{\xi}(2) u_{\xi}(1) \bar{u}(1)+\gamma_{24} u_{\xi}(2) \bar{u}_{\xi}(1) u(1)+\gamma_{25} \bar{u}_{\xi}(2) u_{\xi}(1) u(1)+ \\
& +\gamma_{26} \bar{u}_{\xi}(2) u(2) u(1) .
\end{aligned}
$$

Исключим из уравнения (12) при $j=3$ производные от $u(1)$ по медленным временам $t_{2}$ и $t_{3}$, используя эволюции (9а) при $\sigma=2$ и $\sigma^{\prime}=3$ соответственно, и такие же производные от $u(2)$, используя эволюции (9б) при $\sigma=2$ и $\sigma^{\prime}=3$. Приравняем остающиеся слагаемые почленно, если $\rho_{2} \neq 0$; тогда, обозначив через $R_{i}$ и $I_{i}$ вещественную и мнимую части $\tau_{i}, i=1, \ldots, 12$, получим условия $A_{3}$-интегрируемости в виде

$$
\begin{aligned}
& R_{1}=-\frac{a I_{6}}{4 \rho_{1}}, \quad R_{3}=\frac{(b-a) I_{6}}{2 \rho_{2}}-\frac{a I_{12}}{2 \rho_{2}}, \quad R_{4}=\frac{R_{2}}{2}+\frac{(a-b) I_{6}}{4 \rho_{2}}+\frac{a I_{12}}{4 \rho_{2}}, \\
& R_{5}=\frac{R_{2}}{2}+\frac{(a-b) I_{6}}{4 \rho_{2}}+\frac{(2 b-a) I_{12}}{4 \rho_{2}}, \quad R_{6}=-\frac{a I_{8}}{\rho_{2}}, \quad R_{7}=R_{12}+\frac{(a-b) I_{8}}{\rho_{2}}, \\
& R_{8}=R_{9}=0, \quad R_{10}=R_{12}, \quad R_{11}=R_{12}+\frac{(a-2 b) I_{8}}{\rho_{2}}, \\
& I_{4}=\frac{(b+a) R_{12}}{4 \rho_{2}}+\frac{\rho_{1} I_{1}}{\rho_{2}}+\frac{I_{2}-I_{3}-2 I_{5}}{4}+\frac{\left[2 b(a-b)+a^{2}\right] I_{8}}{4 \rho_{2}^{2}}, \quad I_{7}=0, \\
& I_{9}=2 I_{8}, \quad I_{10}=I_{12}, \quad I_{11}=I_{6}+I_{12} .
\end{aligned}
$$


Для полноты приведем выражения для $\gamma_{j}, j=1, \ldots, 26$, как функций $\tau_{i}, i=$ $1, \ldots, 12$ :

$$
\begin{aligned}
& \gamma_{1}=\frac{3 B}{8 \rho_{1}^{2}}\left[-2 b R_{12}-8 \rho_{1} I_{1}+2\left(I_{2}-2 I_{3}-2 I_{5}\right) \rho_{2}+i(b-5 a) I_{6}+\frac{2 a^{2} I_{8}}{\rho_{2}}-3 i a I_{12}\right], \\
& \gamma_{2}=-\frac{3 B a}{4 \rho_{1}^{2}}\left[i I_{6}+\frac{(a-2 b) I_{8}}{\rho_{2}}+\tau_{12}\right], \quad \gamma_{3}=\frac{3 i B \tau_{3}}{2 \rho_{1}}, \quad \gamma_{4}=0 \\
& \gamma_{5}=\frac{3 i B \tau_{2}}{2 \rho_{1}}, \quad \gamma_{6}=\frac{3 i B \tau_{4}}{\rho_{1}}, \quad \gamma_{7}=\gamma_{5}, \quad \gamma_{8}=\gamma_{3}+\frac{3 i B \tau_{5}}{\rho_{1}} \\
& \gamma_{9}=-\frac{3 B\left(\rho_{2} I_{6}+3 a i I_{8}\right)}{4 \rho_{1}^{2}}, \quad \gamma_{10}=\frac{3 i B \rho_{2} R_{6}}{2 \rho_{1}^{2}}, \quad \gamma_{11}=0, \quad \gamma_{12}=\frac{3 i B \tau_{9}}{2 \rho_{1}} \\
& \gamma_{13}=\frac{3 i B \tau_{11}}{2 \rho_{1}}, \quad \gamma_{14}=0, \quad \gamma_{15}=\frac{3 i B \tau_{12}}{2 \rho_{1}}, \quad \gamma_{16}=\frac{3 i B \tau_{6}}{2 \rho_{1}}, \\
& \gamma_{17}=\gamma_{18}=0, \quad \gamma_{19}=\frac{3 i B \tau_{10}}{2 \rho_{1}}, \quad \gamma_{20}=\gamma_{15}, \quad \gamma_{21}=\frac{3 i B \tau_{8}}{\rho_{1}}, \\
& \gamma_{22}=\gamma_{12}, \quad \gamma_{23}=\gamma_{16}+\gamma_{19}, \quad \gamma_{24}=\gamma_{13}, \quad \gamma_{25}=\frac{3 i B \tau_{7}}{\rho_{1}}, \quad \gamma_{26}=0
\end{aligned}
$$

Условия для случая $C$-интегрируемых или линеаризуемых уравнений аналогичны представленным здесь условиям и их можно найти в работе [26].

\section{4. МНОГОМАСШТАБНЫЕ РАЗЛОЖЕНИЯ ДЛЯ ИСКЛЮЧИТЕЛЬНЫХ МОДЕЛЕЙ}

Выполним многомасштабный анализ дифференциально-разностных моделей (1) для вещественной функции $u_{n}(t)$, где функции $Q_{n}$ заданы в $(2)$. Для этого разложим переменную $u_{n}$ согласно предписаниям из предыдущего раздела и разобьем полученную систему по различным гармоникам и различным степеням $\varepsilon$. Уравнения различных порядков представлены в приложении, а здесь мы только приведем окончательные результаты.

ПреДЛОЖЕНИЕ 4. Все модели (2) удовлетворяют условиям $A_{1}$ - $и A_{2}$-интегрируемости, однако не удовлетворяют условию $A_{3}$-интегрируемости ни при каком выборе соответствующих параметров $\mu_{i}$.

Более того, даже конкретный выбор, сделанный Барашенковым, Окстоби и Пелиновски [1] для случая $Q_{1}$ и $Q_{2}$, является не более интегрируемым, чем остальные. В приложении также показано, что эту нелинейную модель невозможно нетривиально линеаризовать.

\section{5. ЗАКЛЮЧИТЕЛЬНЫЕ ЗАМЕЧАНИЯ}

В настоящей работе с помощью теста на интегрируемость в рамках многомасштабного разложения показано, что набор дискретных $\phi^{4}$-моделей, построенных в работе [1], не является интегрируемым, а имеет лишь некоторую нетривиальную степень интегрируемости, поскольку они удовлетворяют двум самым низшим условиям интегрируемости. 
Модели не удовлетворяют симметриям непрерывной $\phi^{4}$-теории, а лишь обладают бегущими кинковыми решениями. Было бы интересно построить дискретные модели, сохраняющие симметрии непрерывной модели. Это можно проделать [27], однако при этом требуется переменная неравномерная решетка. Поскольку непрерывная модель не является полностью интегрируемой, мы не ожидаем, что такая модель, сохраняющая дискретную симметрию, будет полностью интегрируемой. Представляется интересным также обобщить проделанные в настоящей работе вычисления, а именно выразить многомасштабное разложение в терминах нормальных форм. Таким образом можно было бы использовать полученные результаты для аппроксимации решений исходных уравнений. Исследования ведутся в обоих направлениях.

ПРИЛОЖЕНИЕ

Приведем уравнения, полученные из разбиения уравнения (1) при подстановке вместо поля $u_{n}$ его многомасштабного разложения $(7)$ в различные гармоники и различные порядки по $\varepsilon$. Считается, что уравнения справедливы для любой функции $Q_{n}$, заданной в $(2)$, если специально не оговорено противное.

Порядок в и $\alpha=0$. Получаем

$$
u_{1}^{(0)}=0 .
$$

Порядок $\varepsilon$ и $\alpha=1$. Если потребовать, чтобы $u_{1}^{(1)} \neq 0$, то получим дисперсионное соотношение

$$
\omega^{2}=\frac{4 \sin ^{2}(\kappa h / 2)}{h^{2}}-\frac{1}{2} .
$$

Порядок $\varepsilon^{2}$ и $\alpha=0$. Получаем

$$
u_{2}^{(0)}=0
$$

Порядок $\varepsilon^{2}$ и $\alpha=1$. Учитывая дисперсионное соотношение (19), получаем

$$
\partial_{t_{1}} u_{1}^{(1)}-\alpha_{1} \partial_{n_{1}} u_{1}^{(1)}=0, \quad \alpha_{1} \doteq \frac{\sin (\kappa h)}{h^{2} \omega},
$$

откуда следует, что $u_{1}^{(1)}$ имеет вид

$$
u_{1}^{(1)}=g\left(\xi, t_{j}, j \geqslant 2\right), \quad \xi \doteq h n_{1}+\frac{\sin (\kappa h)}{h \omega} t_{1},
$$

где $g$ - произвольная функция своих аргументов, стремящаяся к нулю при $\xi \rightarrow \pm \infty$.

Порядок $\varepsilon^{2}$ и $\alpha=2$. Учитывая дисперсионное соотношение (19), имеем

$$
u_{2}^{(2)}=0 .
$$

Порядок $\varepsilon^{3}$ и $\alpha=0$. Учитывая соотношение (18), имеем

$$
u_{3}^{(0)}=0
$$

Порядок $\varepsilon^{3}$ и $\alpha=1$. Учитывая дисперсионное соотношение (19) и уравнения (18), (21), получаем

$$
\partial_{t_{1}} u_{2}^{(1)}-\alpha_{1} \partial_{n_{1}} u_{2}^{(1)}=-\partial_{t_{2}} u_{1}^{(1)}-i \rho_{1} \partial_{\xi}^{2} u_{1}^{(1)}-i \rho_{2}\left|u_{1}^{(1)}\right|^{2},
$$




$$
\begin{aligned}
\rho_{1} \doteq & \frac{3+\left(h^{2}-4\right) \cos (\kappa h)+\cos (2 \kappa h)}{2 h^{2} \omega\left[h^{2}-4+4 \cos (\kappa h)\right]} \\
\rho_{2} \doteq & -\frac{4 \mu_{2}+\left(5 \mu_{1}+3 \mu_{3}\right) \cos (\kappa h)+2 \mu_{2} \cos (2 \kappa h)+\mu_{1} \cos (3 \kappa h)}{20 \omega}, \\
\rho_{2} \doteq & -\frac{1}{\omega}\left\{\left(5 \mu_{2}+8 \mu_{3}\right) \mu_{1}-\mu_{2} \mu_{3}+\left[\left(11 \mu_{1}-5 \mu_{2}\right) \mu_{1}+3\left(\mu_{2}+\mu_{3}\right) \mu_{3}\right] \cos (\kappa h)-\right. \\
& \left.-\left(\mu_{1} \mu_{2}+4 \mu_{1} \mu_{3}-2 \mu_{2} \mu_{3}\right) \cos (2 \kappa h)+\mu_{1}\left(\mu_{1}-\mu_{2}\right) \cos (3 \kappa h)\right\}, \\
\rho_{2} \doteq & -\frac{3\left(4+h^{2}\right) \mu_{1}+2 \mu_{2}+3\left(1-2 \mu_{2}\right) \cos (\kappa h)+\left[3\left(4+h^{2}\right) \mu_{1}+4 \mu_{2}\right] \cos (2 \kappa h)}{4 \omega},
\end{aligned}
$$

где (25в) получается в случае модели $Q_{1},\left(25\right.$ г) - в случае модели $Q_{2}$, а $(25$ д) в случае модели $Q_{3}$. Из уравнения (21) следует, что правая часть уравнени (25a) является секулярной. Поэтому необходимо потребовать, чтобы

$$
\begin{gathered}
\partial_{t_{1}} u_{2}^{(1)}-\alpha_{1} \partial_{n_{1}} u_{2}^{(1)}=0 \\
\partial_{t_{2}} u_{1}^{(1)}=-i \rho_{1} \partial_{\xi}^{2} u_{1}^{(1)}-i \rho_{2}\left|u_{1}^{(1)}\right|^{2} \doteq K_{2}\left[u_{1}^{(1)}\right] .
\end{gathered}
$$

Из уравнения (26а) видно, что $u_{2}^{(1)}$ также зависит от $\xi$, в то время как (26б) является интегрируемым НУШ, а из определений (25в)-(25д) нетрудно видеть, что $\rho_{2}$ является вещественным коэффициентом во всех трех случаях. Поэтому все модели являются $A_{1}$-интегрируемыми.

Если потребовать, чтобы наши модели были $A_{1}$-линеаризуемыми [26], то равенство $\rho_{2}=0$ должно выполняться для любого $\kappa$. В этом случае имеем соответственно

$$
\begin{array}{ll}
Q_{1}: & \mu_{1}=\mu_{2}=\mu_{3}=0, \\
Q_{2}: & \mu_{1}=\mu_{3}=0, \\
Q_{3}: & \text { нет решения, }
\end{array}
$$

т. е. в первых двух моделях выбираются только тривиальные линейные случаи, в то время как третья модель не допускает $A_{1}$-линеаризуемой редукции.

Порядок $\varepsilon^{3}$ и $\alpha=2$. Учитывая дисперсионное соотношение (19) и уравнения (18), (23), получаем

$$
u_{3}^{(2)}=0 .
$$

Порядок $\varepsilon^{3}$ и $\alpha=3$. Учитывая дисперсионное соотношение (19), получаем

$$
\begin{aligned}
u_{3}^{(3)} & =\alpha_{2} u_{1}^{(1) 3}, \\
\alpha_{2} & \doteq \frac{h^{2}\left[\mu_{3}+2 \mu_{2} \cos (\kappa h)+2 \mu_{1} \cos (2 \kappa h)\right] \cos (\kappa h)}{20\left[8-2 h^{2}-9 \cos (\kappa h)+\cos (3 \kappa h)\right]} \\
\alpha_{2} & \doteq \frac{h^{2}\left[\mu_{3}+2 \mu_{1} \cos (\kappa h)\right]\left[\mu_{1}-\mu_{2}+\left(\mu_{2}+\mu_{3}\right) \cos (\kappa h)+\mu_{1} \cos (2 \kappa h)\right]}{8-2 h^{2}-9 \cos (\kappa h)+\cos (3 \kappa h)} \\
\alpha_{2} & \doteq \frac{h^{2}\left[\left(4+h^{2}\right) \mu_{1}+2 \mu_{2}+\left(1-2 \mu_{2}\right) \cos (\kappa h)+\left(4+h^{2}\right) \mu_{1} \cos (2 \kappa h)\right]}{4\left[8-2 h^{2}-9 \cos (\kappa h)+\cos (3 \kappa h)\right]}
\end{aligned}
$$

где (29б) получено для случая модели $Q_{1},(29$ в $)$ - для случая модели $Q_{2}$, а $(29$ г) для случая модели $Q_{3}$. 
Порядок $\varepsilon^{4}$ и $\alpha=0$. Учитывая (18), (20), (23), получаем

$$
u_{4}^{(0)}=0 .
$$

Порядок $\varepsilon^{4}$ и $\alpha=1$. Учитывая дисперсионное соотношение (19) и уравнения (18), (21), (26а), (26б), получаем

$$
\begin{aligned}
& \partial_{t_{1}} u_{3}^{(1)}-\alpha_{1} \partial_{n_{1}} u_{3}^{(1)}=-\left(\partial_{t_{3}} u_{1}^{(1)}-K_{3}\left[u_{1}^{(1)}\right]\right)-\left(\partial_{t_{2}} u_{2}^{(1)}-K_{2}^{\prime}\left[u_{1}^{(1)}\right] u_{2}^{(1)}-f_{2}(2)\right), \\
& B \doteq \frac{\left[6-8 h^{2}+h^{4}+2\left(h^{2}-4\right) \cos (\kappa h)+2 \cos (2 \kappa h)\right] \sin (\kappa h)}{6 h^{2} \omega\left[h^{2}-4+4 \cos (\kappa h)\right]^{2}} \\
& K_{2}^{\prime}\left[u_{1}^{(1)}\right] u_{2}^{(1)} \doteq-i \rho_{1}\left[\partial_{\xi}^{2} u_{2}^{(1)}+\frac{\rho_{2}}{\rho_{1}}\left(u_{1}^{(1) 2} \bar{u}_{2}^{(1)}+2\left|u_{1}^{(1)}\right|^{2} u_{2}^{(1)}\right)\right] .
\end{aligned}
$$

В приведенных соотношениях $K_{3}\left[u_{1}^{(1)}\right](11 в)$ представляет собой комплексно-модифицированный КдФ (cmКдФ) поток, второй поток иерархии НУШ, $K_{2}^{\prime}\left[u_{1}^{(1)}\right] u_{2}^{(1)}$ представляет собой производную Фреше по направлению $u_{2}^{(1)}$ потока иерархии НУШ $K_{2}\left[u_{1}^{(1)}\right]$, определяемого соотношением (26б), а $f_{2}(2)$ (13а) является нелинейным вынуждающим членом, зависящим от $u_{1}^{(1)}$ и определяемым коэффициентами $a$ и $b$. Как следствие (21), (26а) правая часть уравнения (31a) является секулярной, так что

$$
\begin{gathered}
\partial_{t_{1}} u_{3}^{(1)}-\alpha_{1} \partial_{n_{1}} u_{3}^{(1)}=0 \\
\partial_{t_{2}} u_{2}^{(1)}-K_{2}^{\prime}\left[u_{1}^{(1)}\right] u_{2}^{(1)}=-\left(\partial_{t_{3}} u_{1}^{(1)}-K_{3}\left[u_{1}^{(1)}\right]\right)+f_{2}(2) .
\end{gathered}
$$

Из уравнения (32а) видно, что $u_{3}^{(1)}$ также зависит от $\xi$, в то время как в силу (26б) первый член в правой части (32б) является секулярным, так что

$$
\begin{aligned}
\partial_{t_{2}} u_{2}^{(1)}-K_{2}^{\prime}\left[u_{1}^{(1)}\right] u_{2}^{(1)} & =f_{2}(2), \\
\partial_{t_{3}} u_{1}^{(1)}-K_{3}\left[u_{1}^{(1)}\right] & =0 .
\end{aligned}
$$

Коэффициенты $a$ и $b$ вынуждающего члена $f_{2}(2)(13 \mathrm{a})$ для модели $Q_{1}$ имеют вид

$$
\begin{aligned}
a= & \frac{1}{\Delta_{a}}\left\{\left[-36\left(h^{2}-4\right) \mu_{1}+3\left(4-8 h^{2}+h^{4}\right) \mu_{2}-5\left(h^{2}-4\right) \mu_{3}\right] \sin (\kappa h)+\right. \\
& +\left[\left(-153+32 h^{2}-4 h^{4}\right) \mu_{1}-6\left(h^{2}-4\right) \mu_{2}-\left(2+8 h^{2}-h^{4}\right) \mu_{3}\right] \sin (2 \kappa h)- \\
& -\left[23\left(h^{2}-4\right) \mu_{1}+\left(36-8 h^{2}+h^{4}\right) \mu_{2}-3\left(h^{2}-4\right) \mu_{3}\right] \sin (3 \kappa h)+ \\
& +\left[\left(-42+8 h^{2}-h^{4}\right) \mu_{1}-3\left(h^{2}-4\right) \mu_{2}+5 \mu_{3}\right] \sin (4 \kappa h)- \\
& \left.-3\left(h^{2}-4\right) \mu_{1} \sin (5 \kappa h)+\mu_{1} \sin (6 \kappa h)\right\}, \\
\Delta_{a}= & 40 \omega\left[h^{2}-4+4 \cos (\kappa h)\right]\left[3+\left(h^{2}-4\right) \cos (\kappa h)+\cos (2 \kappa h)\right], \\
b= & \frac{1}{\Delta_{b}}\left\{12 \mu_{2}+\left(h^{2}-4\right) \mu_{3}+2\left[7 \mu_{1}+\left(h^{2}-4\right) \mu_{2}+5 \mu_{3}\right] \cos (\kappa h)+\right. \\
& \left.+2\left[\left(h^{2}-4\right) \mu_{1}+4 \mu_{2}\right] \cos (2 \kappa h)+6 \mu_{1} \cos (3 \kappa h)\right\} \sin (\kappa h), \\
\Delta_{b}= & 20 \omega\left[h^{2}-4+4 \cos (\kappa h)\right],
\end{aligned}
$$


для модели $Q_{2}$

$$
\begin{aligned}
& a= \frac{1}{\Delta_{a}}\left\{\left\{\left[\left(50-48 h^{2}+7 h^{4}\right) \mu_{2}-46\left(h^{2}-4\right) \mu_{1}+6\left(4-8 h^{2}+h^{4}\right) \mu_{3}\right] \mu_{1}+\right.\right. \\
&\left.+\left[\left(70-21 h^{2}+2 h^{4}\right) \mu_{2}-5\left(h^{2}-4\right) \mu_{3}\right] \mu_{3}\right\} \sin (\kappa h)+ \\
&+\left\{\left[\left(1+10 h^{2}\right) \mu_{2}+\left(-157+16 h^{2}-2 h^{4}\right) \mu_{1}\right] \mu_{1}+\right. \\
&\left.+\left[\left(-58+6 h^{2}+h^{4}\right) \mu_{2}-12\left(h^{2}-4\right) \mu_{1}+\left(-2-8 h^{2}+h^{4}\right) \mu_{3}\right] \mu_{3}\right\} \sin (2 \kappa h)+ \\
&+\left\{-\left[\left(59-19 h^{2}+h^{4}\right) \mu_{2}+17\left(h^{2}-4\right) \mu_{1}+2\left(36-8 h^{2}+h^{4}\right) \mu_{3}\right] \mu_{1}+\right. \\
&\left.+3\left[\left(h^{2}+4\right) \mu_{2}+\left(h^{2}-4\right) \mu_{3}\right] \mu_{3}\right\} \sin (3 \kappa h)+ \\
&+\left\{\left[\left(46-11 h^{2}+h^{4}\right) \mu_{2}-\left(32-8 h^{2}+h^{4}\right) \mu_{1}-6\left(h^{2}-4\right) \mu_{3}\right] \mu_{1}+\right. \\
&\left.+5\left(\mu_{2}+\mu_{3}\right) \mu_{3}\right\} \sin (4 \kappa h)-\left\{\left[\left(13-3 h^{2}\right) \mu_{2}+3\left(h^{2}-4\right) \mu_{1}\right] \mu_{1}+2 \mu_{2} \mu_{3}\right\} \times \\
&\left.\times \sin (5 \kappa h)+\left(\mu_{1}-\mu_{2}\right) \mu_{1} \sin (6 \kappa h)\right\} \\
& \Delta_{a}= 2 \omega\left[h^{2}-4+4 \cos (\kappa h)\right]\left[3+\left(h^{2}-4\right) \cos (\kappa h)+\cos (2 \kappa h)\right], \\
& b= \frac{1}{\Delta_{b}}\left\{\left\{\left[\left(h^{2}-4\right) \mu_{1}-3\left(h^{2}-7\right) \mu_{2}+16 \mu_{3}\right] \mu_{1}+\right.\right. \\
&\left.+\left[\left(h^{2}-8\right) \mu_{2}+\left(h^{2}-4\right) \mu_{3}\right] \mu_{3}\right\} \sin (\kappa h)+ \\
&+\left\{2\left(7 \mu_{1}-6 \mu_{2}\right) \mu_{1}+\left[2\left(h^{2}-4\right) \mu_{1}+\left(13-2 h^{2}\right) \mu_{2}+5 \mu_{3}\right] \mu_{3}\right\} \sin (2 \kappa h)+ \\
&+\left\{\left[\left(h^{2}-4\right) \mu_{1}-\left(h^{2}-5\right) \mu_{2}\right] \mu_{1}+2\left(4 \mu_{1}-3 \mu_{2}\right) \mu_{3}\right\} \sin (3 \kappa h)+ \\
&\left.+3\left(\mu_{1}-\mu_{2}\right) \mu_{1} \sin (4 \kappa h)\right\}, \\
& \Delta_{b}=\omega\left[h^{2}-4+4 \cos (\kappa h)\right],
\end{aligned}
$$

и для модели $Q_{3}$

$$
\begin{aligned}
a= & -\frac{1}{\Delta_{a}}\left\{\left\{4\left(-5+58 \mu_{1}+35 \mu_{2}\right)+\right.\right. \\
& \left.+h^{2}\left[5+\left(26-4 h^{2}+h^{4}\right) \mu_{1}+2\left(2 h^{2}-21\right) \mu_{2}\right]\right\} \sin (\kappa h)+ \\
& +\left[-2+352 \mu_{1}+h^{4}\left(1-22 \mu_{1}-2 \mu_{2}\right)+116 \mu_{2}-4 h^{2}\left(2+3 \mu_{2}\right)\right] \sin (2 \kappa h)- \\
& -\left\{12\left(1+19 \mu_{1}+2 \mu_{2}\right)+h^{2}\left[-3+\left(25-4 h^{2}+h^{4}\right) \mu_{1}+6 \mu_{2}\right]\right\} \sin (3 \kappa h)+ \\
& \left.+\left[5-3\left(h^{4}-16\right) \mu_{1}-10 \mu_{2}\right] \sin (4 \kappa h)+\left[\left(h^{2}+4\right) \mu_{1}+4 \mu_{2}\right] \sin (5 \kappa h)\right\}, \\
\Delta_{a}= & 8 \omega\left[h^{2}-4+4 \cos (\kappa h)\right]\left[3+\left(h^{2}-4\right) \cos (\kappa h)+\cos (2 \kappa h)\right] \\
b= & \frac{1}{\Delta_{b}}\left\{\left[h^{2}\left(1+7 \mu_{1}-2 \mu_{2}\right)+4\left(-1+7 \mu_{1}+4 \mu_{2}\right)\right] \sin (\kappa h)+\left[5+2\left(h^{4}-16\right) \mu_{1}+\right.\right. \\
& \left.\left.+2\left(2 h^{2}-13\right) \mu_{2}\right] \sin (2 \kappa h)+\left[7\left(h^{2}+4\right) \mu_{1}+12 \mu_{2}\right] \sin (3 \kappa h)\right\} \\
\Delta_{b}= & 4 \omega\left[h^{2}-4+4 \cos (\kappa h)\right] .
\end{aligned}
$$

Как можно видеть, во всех трех случаях коэффициенты $a$ и $b$ являются вещественными. Как следствие, все три модели являются $A_{2}$-интегрируемыми.

Порядок $\varepsilon^{4}$ и $\alpha=2$. Учитывая дисперсионное соотношение (19) и уравнения (18), (20), (23), (28), получаем

$$
u_{4}^{(2)}=0 .
$$


Порядок $\varepsilon^{4}$ и $\alpha=3$. Учитывая дисперсионное соотношение (19) и уравнения (19), (22), (29), получаем

$$
u_{4}^{(3)}=\left(\alpha_{3} u_{2}^{(1)}+\alpha_{4} \partial_{\xi} u_{1}^{(1)}\right) u_{1}^{(1) 2}
$$

Поскольку $u_{4}^{(3)}$ не входит в конечный результат, коэффициенты $\alpha_{3}$ и $\alpha_{4}$ здесь явно не выписаны.

Порядок $\varepsilon^{4}$ и $\alpha=4$. Учитывая дисперсионное соотношение (19) и уравнение (23), находим

$$
u_{4}^{(4)}=0 .
$$

Порядок $\varepsilon^{4}$ и $\alpha=0$. Учитывая уравнения (18), (20), (23), (24), (28), получаем

$$
u_{5}^{(0)}=0
$$

Порядок $\varepsilon^{5}$ и $\alpha=1$. Учитывая дисперсионное соотношение (19) и уравнения (18), (20), (21), (23), (26а), (26б), (29), (32а), (33а), (33б), получаем

$$
\begin{aligned}
\partial_{t_{1}} u_{4}^{(1)}-\alpha_{1} \partial_{n_{1}} u_{4}^{(1)}=-\left(\partial_{t_{2}} u_{3}^{(1)}-K_{2}^{\prime}\left[u_{1}^{(1)}\right] u_{3}^{(1)}-f_{2}(3)\right)- & \\
& -\left(\partial_{t_{3}} u_{2}^{(1)}-K_{3}^{\prime}\left[u_{1}^{(1)}\right] u_{2}^{(1)}-f_{3}(2)\right)-\left(\partial_{t_{4}} u_{1}^{(1)}-K_{4}\left[u_{1}^{(1)}\right]\right), \\
C \doteq & \frac{1}{\Delta_{c}}\left\{7\left(5-8 h^{2}+h^{4}\right)+\left(h^{2}-4\right)\left(14-8 h^{2}+h^{4}\right) \cos (\kappa h)+\right. \\
& \left.+\left(28+8 h^{2}-h^{4}\right) \cos (2 \kappa h)+2\left(h^{2}-4\right) \cos (3 \kappa h)+\cos (4 \kappa h)\right\}, \\
\Delta_{c}= & 24 h^{2} \omega\left[h^{2}-4+4 \cos (\kappa h)\right]^{3} .
\end{aligned}
$$

В приведенных соотношениях $K_{4}\left[u_{1}^{(1)}\right](11 г)$ представляет собой третий поток иерархии НУШ, $K_{3}^{\prime}\left[u_{1}^{(1)}\right] u_{2}^{(1)}$ является производной Фреше по направлению $u_{2}^{(1)}$ потока $\operatorname{cmKд\Phi ~} K_{3}\left[u_{1}^{(1)}\right]$, определяемого соотношением $(11 \mathrm{~B})$, а $f_{2}(3)$ и $f_{3}(2)$ - нелинейные вынуждающие члены, определяемые в (13a), (13б). Как следует из (21), (26а), (32а), правая часть уравнения (39a) является секулярной, так что

$$
\begin{aligned}
& \partial_{t_{1}} u_{4}^{(1)}-\alpha_{1} \partial_{n_{1}} u_{4}^{(1)}=0 \\
& \partial_{t_{2}} u_{3}^{(1)}-K_{2}^{\prime}\left[u_{1}^{(1)}\right] u_{3}^{(1)}=-\left(\partial_{t_{3}} u_{2}^{(1)}-K_{3}^{\prime}\left[u_{1}^{(1)}\right] u_{2}^{(1)}-f_{3}(2)\right)- \\
& \quad-\left(\partial_{t_{4}} u_{1}^{(1)}-K_{4}\left[u_{1}^{(1)}\right]\right)+f_{2}(3) .
\end{aligned}
$$

Из первого соотношения видно, что $u_{4}^{(1)}$ также зависит от $\xi$, в то время как в уравнении (40б) в силу (33а) и равенства

$$
\left(\partial_{t_{2}}-K_{2}^{\prime}\left[u_{1}^{(1)}\right]\right) f_{3}(2)=\left(\partial_{t_{3}}-K_{3}^{\prime}\left[u_{1}^{(1)}\right]\right) f_{2}(2)
$$

первый член в правой части является секулярным. Более того, второй член в правой части (40б) также является секулярным, поскольку при приравнивании его нулю получаем обобщенную симметрию для иерархии НУШ, третье уравнение соответствующей иерархии. Поэтому имеем

$$
\begin{aligned}
& \partial_{t_{2}} u_{3}^{(1)}-K_{2}^{\prime}\left[u_{1}^{(1)}\right] u_{3}^{(1)}=f_{2}(3), \\
& \partial_{t_{3}} u_{2}^{(1)}-K_{3}^{\prime}\left[u_{1}^{(1)}\right] u_{2}^{(1)}=-\left(\partial_{t_{4}} u_{1}^{(1)}-K_{4}\left[u_{1}^{(1)}\right]\right)+f_{3}(2) .
\end{aligned}
$$


Наконец, первый член в правой части (41б) является секулярным, поскольку в случае, когда он равен нулю, мы получаем обобщенную симметрию уравнения стКдФ, поскольку оба уравнения принадлежат той же иерархии НУШ. Поэтому получаем

$$
\begin{aligned}
\partial_{t_{3}} u_{2}^{(1)}-K_{3}^{\prime}\left[u_{1}^{(1)}\right] u_{2}^{(1)} & =f_{3}(2), \\
\partial_{t_{4}} u_{1}^{(1)}-K_{4}\left[u_{1}^{(1)}\right] & =0 .
\end{aligned}
$$

Вещественные и мнимые части $\left(R_{j}, I_{j}\right)$ коэффициентов $\tau_{j}, j=1, \ldots, 12$, вынуждающего члена $f_{2}(3)$ независимо от модели имеют вид

$$
\begin{aligned}
& R_{1}=R_{2}=R_{3}=R_{4}=R_{5}=R_{8}=R_{9}=0, \quad R_{10}=R_{12}, \\
& I_{6}=I_{7}=I_{10}=I_{11}=I_{12}=0, \quad I_{9}=2 I_{8} .
\end{aligned}
$$

Далее, для модели $Q_{1}$ имеем

$$
\begin{aligned}
& I_{1}=\frac{1}{20 \rho_{1}^{2} \omega}\left\{2 \alpha_{2} \mu_{2} \rho_{1}^{2}-5\left(2 \rho_{1}^{2} \rho_{2}+3 a B \omega-6 C \rho_{2} \omega\right) \rho_{2}+\alpha_{2} \rho_{1}^{2}\left\{\mu_{2}[3 \cos (2 \kappa h)+\right.\right. \\
& \left.+\cos (4 \kappa h)]+\left[\mu_{3}+2 \mu_{1} \cos (2 \kappa h)\right][2 \cos (\kappa h)+\cos (3 \kappa h)]\right\}, \\
& I_{2}=\frac{2 \mu_{2} \rho_{1}+20\left[(a+2 b) \alpha_{1} \rho_{1}+\left(3 B \alpha_{1}-2 \rho_{1}^{2}+2 C \omega\right) \rho_{2}-3 b B \omega\right]}{20 \rho_{1} \omega}+ \\
& +\frac{6 \mu_{1} \cos (\kappa h)-\mu_{2} \cos (2 \kappa h)-2 \mu_{1} \cos (3 \kappa h)}{20 \omega}, \\
& I_{3}=\frac{2 a \alpha_{1} \rho_{1}+2\left(3 B \alpha_{1}-2 \rho_{1}^{2}+4 C \omega\right) \rho_{2}-3 a B \omega}{2 \rho_{1} \omega}+ \\
& +\frac{\left[4 \mu_{1}+\mu_{3}+3 \mu_{2} \cos (\kappa h)+2 \mu_{1} \cos (2 \kappa h)\right] \cos (\kappa h)}{20 \omega} \\
& I_{4}=\frac{1}{40 \rho_{1} \omega}\left\{2\left[\left(20 b \alpha_{1}+\mu_{2}\right) \rho_{1}+20 C \rho_{2} \omega\right]+\left[\left(5 \mu_{1}+\mu_{3}\right) \cos (\kappa h)+\right.\right. \\
& \left.\left.+\mu_{2} \cos (2 \kappa h)+\mu_{1} \cos (3 \kappa h)\right] \rho_{1}\right\}, \\
& I_{5}=\frac{1}{40 \rho_{1} \omega}\left\{20\left(2 \alpha_{1} \rho_{1}-3 B \omega\right) a-\mu_{2} \rho_{1}+40\left(3 B \alpha_{1}-\rho_{1}^{2}+3 C \omega\right) \rho_{2}+\right. \\
& \left.+2\left[\mu_{2}+2 \mu_{1} \cos (\kappa h)\right] \rho_{1} \cos (2 \kappa h)\right\}, \\
& R_{6}=\frac{\left(2 \alpha_{1} \rho_{1}-3 B \omega\right) \rho_{2}}{\rho_{1} \omega}-\frac{\left[4 \mu_{1}+\mu_{3}+3 \mu_{2} \cos (\kappa h)+2 \mu_{1} \cos (2 \kappa h)\right] \sin (\kappa h)}{10 \omega}, \\
& R_{7}=\frac{20 \alpha_{1} \rho_{2}+\left[\mu_{3}+2 \mu_{1} \cos (2 \kappa h)\right] \sin (\kappa h)+\mu_{2} \sin (2 \kappa h)}{20 \omega}, \\
& I_{8}=\frac{\left(5 \mu_{1}+3 \mu_{3}\right) \cos (\kappa h)+2[2+\cos (2 \kappa h)] \mu_{2}+\mu_{1} \cos (3 \kappa h)}{20 \omega}, \\
& R_{10}=\frac{\left(2 \alpha_{1} \rho_{1}-3 B \omega\right) \rho_{2}}{\rho_{1} \omega}-\frac{\left[4 \mu_{1}+\mu_{3}+3 \mu_{2} \cos (\kappa h)+2 \mu_{1} \cos (2 \kappa h)\right] \sin (\kappa h)}{10 \omega}, \\
& R_{11}=\frac{20 \alpha_{1} \rho_{2}+\left[\mu_{3}+2 \mu_{1} \cos (2 \kappa h)\right] \sin (\kappa h)+\mu_{2} \sin (2 \kappa h)}{10 \omega},
\end{aligned}
$$


для модели $Q_{2}$

$$
\begin{aligned}
& I_{1}=\frac{1}{4 \rho_{1}^{2} \omega}\left\{4\left(3 \mu_{1} \mu_{2}+4 \mu_{1} \mu_{3}-\mu_{2} \mu_{3}\right) \alpha_{2} \rho_{1}^{2}-\left(2 \rho_{1}^{2} \rho_{2}+3 a B \omega-6 C \rho_{2} \omega\right) \rho_{2}+\right. \\
& +4 \alpha_{2} \rho_{1}^{2}\left\{\left[\left(7 \mu_{1}-2 \mu_{2}\right) \mu_{1}+2\left(\mu_{2}+\mu_{3}\right) \mu_{3}\right] \cos (\kappa h)+\right. \\
& +3 \mu_{1}\left(\mu_{2}+2 \mu_{3}\right) \cos (2 \kappa h)+\left[\left(4 \mu_{1}-3 \mu_{2}\right) \mu_{1}+\left(\mu_{2}+\mu_{3}\right) \mu_{3}\right] \cos (3 \kappa h)+ \\
& \left.\left.+\left(\mu_{1}-\mu_{2}\right)\left[2 \mu_{3} \cos (4 \kappa h)+\mu_{1} \cos (5 \kappa h)\right]\right\}\right\} \text {, } \\
& I_{2}=\frac{(a+2 b) \alpha_{1} \rho_{1}+\left(3 B \alpha_{1}-2 \rho_{1}^{2}+2 C \omega\right) \rho_{2}-3 b B \omega+2\left(\mu_{2}+2 \mu_{3}\right) \mu_{1} \rho_{1}}{\rho_{1} \omega}+ \\
& +\frac{2\left\{3 \mu_{1}^{2} \cos (\kappa h)+\left(\mu_{2}-\mu_{1}\right)\left[\mu_{3} \cos (2 \kappa h)+\mu_{1} \cos (3 \kappa h)\right]\right\}}{\omega}, \\
& I_{3}=\frac{1}{2 \rho_{1} \omega}\left\{2 a \alpha_{1} \rho_{1}+2\left(3 B \alpha_{1}-2 \rho_{1}^{2}+4 C \omega\right) \rho_{2}-3 a B \omega\right\}+ \\
& +\frac{1}{\omega}\left\{2\left(3 \mu_{1}-\mu_{2}\right) \mu_{1}+\left(\mu_{2}+\mu_{3}\right) \mu_{3}+2\left[\left(\mu_{2}+3 \mu_{3}\right) \mu_{1}-\mu_{2} \mu_{3}\right] \cos (\kappa h)+\right. \\
& \left.+2\left(\mu_{1}-\mu_{2}\right) \mu_{1} \cos (2 \kappa h)\right\} \cos (\kappa h), \\
& I_{4}=\frac{1}{2 \rho_{1} \omega}\left\{2\left[b \alpha_{1}+\left(\mu_{2}+2 \mu_{3}\right) \mu_{1}\right] \rho_{1}+2 C \rho_{2} \omega+\left\{\left[\left(7 \mu_{1}-3 \mu_{2}\right) \mu_{1}+\right.\right.\right. \\
& \left.\left.\left.+\left(\mu_{2}+\mu_{3}\right) \mu_{3}\right] \cos (\kappa h)+\left(\mu_{1}-\mu_{2}\right)\left[2 \mu_{3} \cos (2 \kappa h)+\mu_{1} \cos (3 \kappa h)\right]\right\} \rho_{1}\right\}, \\
& I_{5}=\frac{1}{2 \rho_{1} \omega}\left\{6\left(B \alpha_{1}+C \omega\right) \rho_{2}-2\left[\left(\mu_{1}-\mu_{2}\right) \mu_{3}+\rho_{1} \rho_{2}\right] \rho_{1}+\left(2 \alpha_{1} \rho_{1}-3 B \omega\right) a+\right. \\
& \left.+2\left[\left(\mu_{1}+2 \mu_{2}\right) \cos (\kappa h)+\left(\mu_{2}+2 \mu_{3}\right) \cos (2 \kappa h)+\left(\mu_{1}-\mu_{2}\right) \cos (3 \kappa h)\right] \mu_{1} \rho_{1}\right\}, \\
& R_{6}=\frac{1}{\rho_{1} \omega}\left\{\left(2 \alpha_{1} \rho_{1}-3 B \omega\right) \rho_{2}-2\left\{2\left(3 \mu_{1}-\mu_{2}\right) \mu_{1}+\left(\mu_{2}+\mu_{3}\right) \mu_{3}+\right.\right. \\
& \left.\left.+2\left[\left(\mu_{2}+3 \mu_{3}\right) \mu_{1}-\mu_{2} \mu_{3}\right] \cos (\kappa h)+2 \mu_{1}\left(\mu_{1}-\mu_{2}\right) \cos (2 \kappa h)\right\} \rho_{1} \sin (\kappa h)\right\}, \\
& R_{7}=\frac{1}{\omega}\left\{\alpha_{1} \rho_{2}+\left[\left(\mu_{1}-3 \mu_{2}\right) \mu_{1}+\left(\mu_{2}+\mu_{3}\right) \mu_{3}\right] \sin (\kappa h)+\right. \\
& \left.+\left(\mu_{1}-\mu_{2}\right)\left[2 \mu_{3} \sin (2 \kappa h)+\mu_{1} \sin (3 \kappa h)\right]\right\}, \\
& I_{8}=\frac{1}{\omega}\left\{\left(5 \mu_{2}+8 \mu_{3}\right) \mu_{1}-\mu_{2} \mu_{3}+\left[\left(11 \mu_{1}-5 \mu_{2}\right) \mu_{1}+3\left(\mu_{2}+\mu_{3}\right) \mu_{3}\right] \cos (\kappa h)+\right. \\
& \left.+\left[\left(\mu_{2}+4 \mu_{3}\right) \mu_{1}-2 \mu_{2} \mu_{3}\right] \cos (2 \kappa h)+\left(\mu_{1}-\mu_{2}\right) \mu_{1} \cos (3 \kappa h)\right\}, \\
& R_{10}=\frac{1}{\rho_{1} \omega}\left\{\left(2 \alpha_{1} \rho_{1}-3 B \omega\right) \rho_{2}-2\left\{2\left(3 \mu_{1}-\mu_{2}\right) \mu_{1}+\left(\mu_{2}+\mu_{3}\right) \mu_{3}+\right.\right. \\
& \left.\left.+2\left[\left(\mu_{2}+3 \mu_{3}\right) \mu_{1}-\mu_{2} \mu_{3}\right] \cos (\kappa h)+2\left(\mu_{1}-\mu_{2}\right) \mu_{1} \cos (2 \kappa h)\right\} \rho_{1} \sin (\kappa h)\right\}, \\
& R_{11}=\frac{1}{\omega} 2\left\{\alpha_{1} \rho_{2}+\left[\left(\mu_{1}-3 \mu_{2}\right) \mu_{1}+\left(\mu_{2}+\mu_{3}\right) \mu_{3}\right] \sin (\kappa h)+\right. \\
& \left.+\left(\mu_{1}-\mu_{2}\right)\left[2 \mu_{3} \sin (2 \kappa h)+\mu_{1} \sin (3 \kappa h)\right]\right\}
\end{aligned}
$$


и для модели $Q_{3}$

$$
\begin{aligned}
& I_{1}=\frac{1}{4 \rho_{1}^{2} \omega}\left\{\left[\left(h^{2}+4\right) \mu_{1}+2 \mu_{2}\right] \alpha_{2} \rho_{1}^{2}-\left(2 \rho_{1}^{2} \rho_{2}+3 a B \omega-6 C \rho_{2} \omega\right) \rho_{2}+\right. \\
& +\alpha_{2} \rho_{1}^{2}\left\{2\left(1-2 \mu_{2}\right) \cos (\kappa h)+3\left(h^{2}+4\right) \mu_{1} \cos (2 \kappa h)+\left(1-2 \mu_{2}\right) \cos (3 \kappa h)+\right. \\
& \left.\left.+\left[\left(h^{2}+4\right) \mu_{1}+2 \mu_{2}\right] \cos (4 \kappa h)\right\}\right\} \text {, } \\
& I_{2}=\frac{(a+2 b) \alpha_{1} \rho_{1}+\left(3 B \alpha_{1}-2 \rho_{1}^{2}+2 C \omega\right) \rho_{2}-3 b B \omega}{\rho_{1} \omega}+ \\
& +\frac{\left(h^{2}+4\right) \mu_{1}-4 \rho_{1} \rho_{2}-\left[\left(h^{2}+4\right) \mu_{1}+2 \mu_{2}\right] \cos (2 \kappa h)}{2 \omega}, \\
& I_{3}=\frac{2 a \alpha_{1} \rho_{1}+2\left(3 B \alpha_{1}-2 \rho_{1}^{2}+4 C \omega\right) \rho_{2}-3 a B \omega}{2 \rho_{1} \omega}+ \\
& +\frac{\left\{1-2 \mu_{2}+4\left[\left(h^{2}+4\right) \mu_{1}+\mu_{2}\right] \cos (\kappa h)\right\} \cos (\kappa h)}{4 \omega}, \\
& I_{4}=\frac{1}{8 \rho_{1} \omega}\left\{2\left[4 b \alpha_{1}+\left(h^{2}+4\right) \mu_{1}\right] \rho_{1}+8 C \rho_{2} \omega+\left\{\left(1-2 \mu_{2}\right) \cos (\kappa h)+\right.\right. \\
& \left.\left.+2\left[\left(h^{2}+4\right) \mu_{1}+2 \mu_{2}\right] \cos (2 \kappa h)\right\} \rho_{1}\right\} \text {, } \\
& I_{5}=\frac{1}{4 \rho_{1} \omega}\left\{12\left(B \alpha_{1}+C \omega\right) \rho_{2}-\left[\left(h^{2}+4\right) \mu_{1}+2\left(\mu_{2}+2 \rho_{1} \rho_{2}\right)\right] \rho_{1}+\right. \\
& \left.+2\left(2 \alpha_{1} \rho_{1}-3 B \omega\right) a+\left(h^{2}+4\right) \mu_{1} \rho_{1} \cos (2 \kappa h)\right\}, \\
& R_{6}=\frac{\left(2 \alpha_{1} \rho_{1}-3 B \omega\right) \rho_{2}}{\rho_{1} \omega}+\frac{\left(2 \mu_{2}-1\right) \sin (\kappa h)-2\left[\left(h^{2}+4\right) \mu_{1}+\mu_{2}\right] \sin (2 \kappa h)}{2 \omega} \text {, } \\
& R_{7}=\frac{4 \alpha_{1} \rho_{2}+\left(1-2 \mu_{2}\right) \sin (\kappa h)+2\left[\left(h^{2}+4\right) \mu_{1}+2 \mu_{2}\right] \sin (2 \kappa h)}{4 \omega}, \\
& I_{8}=\frac{3\left(h^{2}+4\right) \mu_{1}+2 \mu_{2}+3\left(1-2 \mu_{2}\right) \cos (\kappa h)+\left[3\left(h^{2}+4\right) \mu_{1}+4 \mu_{2}\right] \cos (2 \kappa h)}{4 \omega}, \\
& R_{10}=\frac{\left(2 \alpha_{1} \rho_{1}-3 B \omega\right) \rho_{2}}{\rho_{1} \omega}-\frac{\left(1-2 \mu_{2}\right) \sin (\kappa h)+2\left[\left(h^{2}+4\right) \mu_{1}+\mu_{2}\right] \sin (2 \kappa h)}{2 \omega}, \\
& R_{11}=\frac{4 \alpha_{1} \rho_{2}+\left(1-2 \mu_{2}\right) \sin (\kappa h)+2\left[\left(h^{2}+4\right) \mu_{1}+2 \mu_{2}\right] \sin (2 \kappa h)}{2 \omega} .
\end{aligned}
$$

Поскольку коэффициенты (44)-(46) не удовлетворяют алгебраическим соотношениям (16), для любого $Q A_{3}$-интегрируемость никогда не имеет места.

Благодарности. Настоящая работа выполнена при частичной финансовой поддержке Министерства образования и науки Италии, PRIN "Непрерывные и дискретные нелинейные интегрируемые эволюции: от волн на воде до симплектических отображений".

\section{Список литературы}

[1] I. V. Barashenkov, O. F. Oxtoby, D. E. Pelinovsky, Phys. Rev. E, 72:3 (2005), 035602, 4 pp., arXiv: nlin/0506007.

[2] A. R. Bishop, T. Schneider (eds.), Solitons and Condensed Matter Physics, Springer Ser. Solid-State Sci., 8, Springer, Berlin, 1978. 
[3] R. Rajaraman, Solitons and Instantons, North-Holland, Amsterdam, 1982.

[4] M. J. Rice, A. R. Bishop, J. A. Krumhansl, S. E. Trullinger, Phys. Rev. Lett., 36:8 (1976), 432-435; W. P. Su, J. R. Schrieffer, A. J. Heeger, Phys. Rev. B, 22:4 (1980), 2099-2111; H. Morikawa, I. Matsuda, S. Hasegawa, Phys. Rev. B, 70:8 (2004), 085412, 6 pp.

[5] J. F. Currie, A. Blumen, M. A. Collins, J. Ross, Phys. Rev. B, $19: 7$ (1979), 3645-3655.

[6] S. V. Dmitriev, T. Shigenari, A. A. Vasiliev, K. Abe, Phys. Rev. B, 55:13 (1997), 8155-8164.

[7] I. Chochliouros, J. Pouget, J. Phys., 7:46 (1995), 8741-8756.

[8] V.M. Karpan, Y. Zolotaryuk, P.L. Christiansen, A. V. Zolotaryuk, Phys. Rev. E, 70:5 (2004), 056602, 11 pp.

[9] J. A. Combs, S. Yip, Phys. Rev. B, 28:12 (1983), 6873-6885.

[10] P. Prelovek, I. Sega, J. Phys. C, 14:36 (1981), 5609-5614.

[11] S. Flach, Y. Zolotaryuk, K. Kladko, Phys. Rev. E, 59:5 (1999), 6105-6115, arXiv: patt-sol/9812004.

[12] P. G. Kevrekidis, Physica D, 183:1-2 (2003), 68-86.

[13] Yu. S. Kivshar, A. Sänchez, L. Väzquez, Phys. Rev. A, 45:2 (1992), 1207-1212; S. Flach, C. R. Willis, Phys. Rev. E, 47:6 (1993), 4447-4456; S. Flach, K. Kladko, Phys. Rev. E, 54:3 (1996), 2912-2916, arXiv: cond-mat/9709069; J. C. Comte, P. Marquié, M. Remoissenet, Phys. Rev. E, 60:6 (1999), 7484-7489; P. Maniadis, G. P. Tsironis, A. R. Bishop, A. V. Zolotaryuk, Phys. Rev. E, 60:6 (1999), 7618-7621; P. G. Kevrekidis, M. I. Weinstein, Physica D, 142:1-2 (2000), 113-152, arXiv: nlin/0003006; A. B. Adib, C. A. S. Almeida, Phys. Rev. E, 64:3 (2001), 037701, 4 pp., arXiv: hep-th/0104225.

[14] J. M. Speight, R. S. Ward, Nonlinearity, 7:2 (1994), 475-484, arXiv: patt-sol/9911008.

[15] J. M. Speight, Nonlinearity, 10:6 (1997), 1615-1625, arXiv: patt-sol/9703005.

[16] J. M. Speight, Nonlinearity, 12:5 (1999), 1373-1387, arXiv: hep-th/9812064.

[17] D. Levi, C. Scimiterna, Appl. Anal., 89:4 (2010), 507-527.

[18] C. Scimiterna, Multiscale techniques for nonlinear difference equations, Ph.D. thesis, Roma Tre University, 2009, http://dspace-roma3.caspur.it/handle/2307/408.

[19] D. Levi, J. Phys. A, 38:35 (2005), 7677-7689, arXiv: nlin/0505061.

[20] F. Nijhoff, H. Capel, Acta Appl. Math., 39:1-3 (1995), 133-158.

[21] A. Ramani, частное сообщение, 2006.

[22] C. Viallet, частное сообщение, 2006.

[23] A. Degasperis, S. V. Manakov, P. M. Santini, Physica D, 100:1-2 (1997), 187-211.

[24] Y. Kodama, A. V. Mikhailov, "Obstacles to asymptotic integrability", Algebraic Aspects of Integrable Systems, Progr. Differ. Equ. Appl., 26, Birkhäuser, Boston, MA, 1997, 173-204; Y. Hiraoka, Y. Kodama, "Normal form and solitons", Integrability, Lecture Notes in Phys., 767, ed. A. V. Mikhailov, Springer, Berlin, 2009, 175-214.

[25] A. Degasperis, M. Procesi, "Asymptotic integrability", Symmetry and Perturbation Theory, eds. A. Degasperis, G. Gaeta, World Sci., Singapore, 1999, 23-37; A. Degasperis, "Multiscale expansion and integrability of dispersive wave equations", Integrability, ed. A. V. Mikhailov, Springer, Berlin, 2009, 215-244.

[26] C. Scimiterna, D. Levi, SIGMA, 6 (2010), 070, 17 pp.

[27] D. Levi, P. Winternitz, J. Phys. A, 39:2 (2006), R1-R63, arXiv: nlin/0502004. 